\title{
A multilayered healthcare architecture using relational trust network
}

\author{
Somyajit Chakraborty ${ }^{1}$ and Avijit Gayen ${ }^{2}$
}

Department of Computer Science and Engineering, Techno India University

\footnotetext{
${ }^{1}$ somyajit.ch@breadandcode.online
}

2 avijit.g@technoindiaeducation.com 


\begin{abstract}
Medical Trust-Network is one of the most promising fields of study in network science. Establishment of trust within medical entities ensures better treatment and increases better medical facilities. The word 'Trust' signifies a very important behavioral aspect between any human entities, especially among doctors and patients. To represent such relationships Trust Network Models are built to express the interactions between human entities within such networks. Though the idea of a Trust-Network has traditionally been one of the major areas of research, yet the concept of a medical trust network model is relatively a new domain. In this paper, we introduce an overall multilayered Trust Network to represent the entire healthcare architecture. More specifically our model is based on an evolutionary graph system with a discrete relationship between the three most important entities of any healthcare system, namely - Doctors, Departments, and Hospitals. Observations indicate that based on our model, the medical healthcare system is a multilayered model unlike a feed-forward model as indicated by previous studies.
\end{abstract}

Keywords:Healthcare system, medical network, trust, mathematical modeling, complex network 


\section{Introduction}

In the field of medical and healthcare services, 'Doctors' play a very crucial role who can lead the medical community with their research expertise and clinic practices. The doctors in the medical community are connected with other doctors through their workplace clinics or hospitals and their shared departments. Thus, altogether doctors, hospitals and their departments forms a huge medical social network. Like any other social network 'Trust' plays a very important role in defining this network. Studies have shown that patients tend to choose doctors based on the number of workplaces or hospitals they have worked in and also type of department [1]. So, trust is key element in establishing a medical network using doctors, hospitals and their departments. Before establishing such network, let us first start with the definition of trust.

'Trust' is the measure of willingness to believe in a user based on its competence (e.g. goodness, strength, ability) and behavior within a specific context at a given time. It is a directional relationship from the trustor - the user that evaluates its trust on the target user - to the trustee - the user that is the target of the trust evaluation. According to Yuan et al. 2011 Trust is transitive, so if A trusts B and B trusts C, A will trust C to some extent. This enables the trust propagations between users. Trust network is therefore constructed: the users act as the nodes and their trusts act as the edges [2]. Another definition of 'Trust', given by Levent et al. 2013, is the subjective probability that another agent will act in accordance with one's interests. It is a complex concept that may incorporate a plethora of distinct concepts such as the convergence of interests, compatibility of incentives, competence, and knowledge. Often, it is an aggregate of all of those concepts [3].

Trust-based complex networks have been used in a plethora of applications[4] such as recommender systems[5], productivity assessments[6], and security mechanisms[7]. Establishments of various trust network models in collaboration networks $[\mathbf{8}][\mathbf{9}]$, social ego networks $[\mathbf{9}]$, and likewise, has opened various evolutionary multi-layered complex networks to model trust between distinct or similar entities. The first leap towards modeling such trust networks came with modeling of the scale-free[10] www or the world wide web[11][12]. One of the most promising fields which require a trust network model is the relationships among medical entities like doctors, departments, and various hospitals or medical centers.

Today's healthcare services rely on popularity based assessment models[13][14], which undermines various good medical centers or doctors. Relationships between health care providers are essential to a functioning health care system. Physicians rely on their relationships with physician colleagues for patient referrals (Gonzalez and Rizzo 1991)[15], clinical advice (Keating, Zaslavsky, and Ayanian 1998)[16], and information about the latest clinical advances (Gabbay and le May 2004)[17]. Another reason for establishing a network between various medical entities is to study the strength of the medical system and the flow of information or data among the medical entities(here, doctors and hospitals). One of the major hurdles while establishing such said Trust-Networks is the lack of data on the relationships between various doctors or medical center tie-ups[18]. One way to neutralize this problem is to conduct surveys to collect the needed information to establish relationships between doctors, but such de novo survey work requires a lot of manual data collection which is often inefficient.

In this paper, we introduce a medical trust-network that is very detailed encompassing three 
important medical entities - doctors, departments, and hospitals. Our model also efficiently negates the problem with the lack of data between different medical entities which we will discuss in the following sections.

\section{Related Work}

Trust Networks in healthcare services, though relatively a new approach, still there is considerable foundational work in this field. Traditionally 'Trust' has been a key role in assessing health care services, more specifically as a performance indicator [19]. The first apt comparative study on the role of trust affecting physician-physician relationship as well as the patient-physician relationship was studied in early 2000s by Pearson and Raeke. Their work incorporated a synopsis of theories about patient trust and the evolution of methods to measure it [20]. In their paper, they pointed out the problem of lack of data which we have discussed in the previous section.

The next study incorporates the role of public trust for assessing the expertise of medical professionals. Their statistical analysis by univariable linear regression of the specific determinants of generic assessments of public trust (confidence) suggested that the key aspects were patient centred care and levels of professional expertise. Being covered by private health insurance was also a key determinant of levels of public trust [21]. But like the previous studies the lack of data was still a great challenge for the study and the concentration on only the medical professionals and patients did not encompass the entire medical healthcare structure. A referral based network study [22][23] shows that the patients are referred by doctors within their personal networks. This study pointed out that despite the referral system of patients being conducted within a known network of doctors there are factors like workplace influence like hospitals is also a key factor influencing the referral system.

In [18], Barnett et al. proposed a mapping for the network of physicians with the help of administrative data using a small-world network analysis proposed by Newman in 2003 [24]. The study provided a detailed complex network of the physician networks, but the problem was the lack of external factors like hospitals or departments which influences this evolutionary network model. Their study indicated that a key factor influencing the entire physician network is the number of patients these physicians are sharing. In [25], the authors confirmed that sharing patients is indeed a key factor influencing the medical doctor networks, but they also pointed out the workplace rivalry as well as departments are also key players in a medical network. Social Network Analysis (SNA) and ego mining studies [26][27] have shown that social media can be used to negate the problem of lack of data about physicians or hospitals. The detailed overall network of medical entities like hospitals, doctors, and departments was presented by Guo et al. in [28], which provided the foundational groundwork for establishment of any complex network of these said entities. Their study was based primarily on opinion leader based study [30], which made their study include only the key doctors, instead of all the doctors in any medical healthcare system. They did not explore the Trust-Network specifically in their work, as they primarily concentrated on establishing a recommender system.

The closest detailed network of the medical healthcare system was proposed by Guo et al. which consisted of a patient-centered model with a network study for each individual medical 
entity like hospitals, doctors, and their departments [28]. Though they laid a foundation to a complex medical network, they also failed to explore the inter-relation between these medical entities, which can be explored by only a multilayered network model. We have already described a basic relational or discrete overview of trust in medical system in our study previously [29]. The novelty of this work is to propose a multilayered network model with a simple discrete relationship incorporating all the entities in a well built medical TrustNetwork.

In the next sections, we describe our method for framing a generalised multilayered trust network in the medical healthcare system.

\section{Proposed Network Model}

In this paper, we propose a multilayered complex network to engineer the Trust-Network among three different medical entities, namely, 1. Hospitals 2. Doctors and 3. Departments. Before we go into developing a Trust-Network System we shall establish a network system where Multiple related entities can be graphed such that an elegant mathematical relation holds true. For that, we shall analyze and mathematically formulate a Graph Network System whose nodes are influenced by edge parameters. Since specifically we understand that a Graph Network System could consist of multiple parameters, therefore a need arises to define a multi-layer Network System where each layer has graphs whose nodes have relations with edge parameters.

\section{Relational Network Theorems}

In this section, we shall provide the foundational mathematical theorems on which our Trust-Network for medical system shall be built. With the proposed three theorems and its corollaries we shall visualize the Trust-Network as Relational Network System in each layer of larger Relational Multilayered Network. If we consider Graphs whose edges are influenced by parameters that are related or corresponds to its nodes then we shall call such graphs as Relational Network Systems. Now, if such graphs are the layers of a bigger Multilayered Network Systems then by a sense of logic we will find that nodes of each and every graph of different layers are related to one another and so we shall call such networks as Relational Multi-layer Network Systems. In the course of our study we shall denote our multi-layer network as $\mathcal{M}=(\boldsymbol{Y}, \mathfrak{J})$ and within the network we shall denote layers as $G_{\alpha}$ as layer where $\alpha \in\{1, . ., M\}$. The key notations that shall be used to define our network model is given in table 1. Now we shall establish the properties of our Trust Network model.

Lemma 1. For a observable set of parameters between any two nodes in any layer given as $X_{i}^{\alpha}$ and $X_{j}^{\alpha}$ the relation:

$$
n\left(X_{i}^{\alpha} \cap X_{j}^{\alpha}\right)=n\left(X_{i}^{\alpha}\right)+n\left(X_{j}^{\alpha}\right)-n\left(X_{i}^{\alpha} \cup X_{j}^{\alpha}\right)
$$

holds true where $\alpha \in\{1, . ., M\}$. 
Table 1: Notations

\begin{tabular}{ll}
\hline $\mathcal{M}$ & The Multi-layer Network \\
$X_{i / j}^{\alpha}$ & Any node i/j in layer $\alpha$ \\
$\left\{X_{i / j}^{\alpha}\right\}$ & Set of parameters of node $X_{i / j}^{\alpha}$ \\
$X_{\alpha}$ & Nodes of layer $\alpha$ network \\
$W_{\alpha}$ & Weight of edges of layer $\alpha$ network \\
$P_{\alpha}$ & Total set(union) of parameters of nodes of layer $\alpha$ network \\
$W_{i j}^{\alpha}$ & Weight of the edge between $X_{i}^{\alpha}$ and $X_{j}^{\alpha}$ \\
$A^{[\alpha]}$ & Adjecency matrix of nodes of layer $\alpha$ network \\
$E_{i j}^{\alpha \beta}$ & Inter-layer edge between node $X_{i}^{\alpha}$ of layer $\alpha$ network and node $X_{j}^{\beta}$ \\
$W_{i j}^{\alpha \beta}$ & of layer $\beta$ network \\
$A^{[\alpha, \beta]}$ & Inter-layer edge weight between node $X_{i}^{\alpha}$ of layer $\alpha$ network and \\
$C_{i / j}^{\alpha / \beta}$ & node $X_{j}^{\beta}$ of layer $\beta$ network \\
\hline
\end{tabular}

Proof 1. By the formula for any arbitrary sets A and B the total number of observable items between them is given by the relation $n(A \cup B)=n(A)+n(B)-n(A \cap B)$, where $n(A \cap B)$ gives the number of similar items between sets $\mathrm{A}$ and $\mathrm{B}$. Using this simple relation we can conversely write $n(A \cap B)=n(A)+n(B)-n(A \cup B)$. Now consider the arbitrary nodes $X_{i}^{\alpha}$ and $X_{j}^{\alpha}$ which are in the same layer $G_{\alpha}$, the similar parameters shared by these two nodes must be given by the relation $n\left(X_{i}^{\alpha} \cap X_{j}^{\alpha}\right)=n\left(X_{i}^{\alpha}\right)+n\left(X_{j}^{\alpha}\right)-n\left(X_{i}^{\alpha} \cup X_{j}^{\alpha}\right)$ where $\alpha \in\{1, . ., M\}$ and $1 \leq i, j \leq N_{\alpha}$.

Lemma 2. For a set system where $\mathrm{P}$ represents the overall set of parameters and $A, B \subset P$ then the $\operatorname{rank}(\mathrm{R})$ or the best score between $\mathrm{A}$ and $\mathrm{B}$ should be given by

$$
R=n(P)-n(A \cap B)
$$

Proof 2. The best rank between any two arbitrary sets A and B must have a low value if they have greater similarity. So based on better similarity the rank of their relation can be determined. In Lemma 1 we have already established that the similarity between $\mathrm{A}$ and $\mathrm{B}$ is given as $n(A \cap B)$. Now we now that $A, B \subset P$ and $\mathrm{P}$ is the total set of parameters, therefore in the relation $R=n(P)-n(A \cap B)$ the value of $\mathrm{R}$ decreases if the similarity between $\mathrm{A}$ and $\mathrm{B}$ is more. Thus $\mathrm{R}$ is the rank of the relation between any two arbitrary sets.

Theorem 1. In a layer $G_{\alpha}=\left(X_{\alpha}, W_{\alpha}\right)$, where $X_{\alpha}$ are the nodes and $W_{\alpha}$ are the edge weights and $P_{\alpha}$ is the set of total parameters of the $X_{\alpha}$, then between any two nodes in the layer the relation:

$$
W_{i j}^{\alpha}=n\left(P_{\alpha}\right)-n\left(X_{i}^{\alpha} \cap X_{j}^{\alpha}\right)
$$


holds true where, $W_{i j}^{\alpha} \in W_{\alpha}$ and $i \neq j$ and the nodes $X_{i}^{\alpha}, X_{j}^{\alpha} \in P_{\alpha}$.

Corollary 1.1 In a layer $G_{\alpha}=\left(X_{\alpha}, E_{\alpha}\right)$, where $X_{\alpha}$ are the nodes and $E_{\alpha}$ are the edges, then $E_{\alpha}=\left\{\left(X_{i}^{\alpha}, X_{j}^{\alpha}\right) \mid\left(X_{i}^{\alpha}, X_{j}^{\alpha}\right) \in\left(\begin{array}{c}X_{\alpha} \\ 2\end{array}\right), i \neq j\right\}$ if the graph is unweighted.

Corollary 1.2 In a layer $G_{\alpha}=\left(X_{\alpha}, E_{\alpha}\right)$, where $X_{\alpha}$ are the nodes and $E_{\alpha}$ are the edges and $W_{\alpha}$ are the edge weights, then the adjacency matrix of layer $G_{\alpha}$ is given by $A^{[\alpha]}=\left(a_{i j}^{\alpha}\right) \in$ $\Re^{N_{\alpha} \times N_{\alpha}}$ where,

$$
\left(a_{i j}^{\alpha}\right)=\left\{\begin{array}{cc}
1 & , \text { if }\left(X_{i}^{\alpha}, X_{j}^{\alpha}\right) \in E_{\alpha} \\
0 & , \text { otherwise }
\end{array}\right.
$$

and $1 \leq i, j \leq N_{\alpha}$ and $1 \leq \alpha \leq M$.

Proof 3. In layer $G_{\alpha}$ we have the graph structure $G_{\alpha}=\left(X_{\alpha}, W_{\alpha}\right)$, where $X_{\alpha}$ are the nodes and is given by $X_{\alpha}=\left\{X_{1}^{\alpha}, \ldots, X_{N_{\alpha}}^{\alpha}\right\}$ and the $W_{\alpha}$ are the weights. From Lemma 1 we can calculate the value of similar items possessed by any two arbitrary nodes of $G_{\alpha}$ from the expression $n\left(X_{i}^{\alpha} \cap X_{j}^{\alpha}\right)$, where $1 \leq i, j \leq N_{\alpha}$ and $1 \leq \alpha \leq M$. Now the term $n\left(X_{i}^{\alpha} \cap X_{j}^{\alpha}\right)$ will be greater if $X_{i}^{\alpha}$ and $X_{j}^{\alpha}$ share more items or parameters between them. But for the rank between $X_{i}^{\alpha}$ and $X_{j}^{\alpha}$ we need the least score between these nodes. We have the total set of parameters $P_{\alpha}$ of layer $G_{\alpha}$, then the following relation has to be true:

$$
\bigcup_{k=1}^{N_{\alpha}} X_{k}^{\alpha} \subseteq P_{\alpha}
$$

since, $P_{\alpha}$ is the universal set of parameters of the nodes in layer $G_{\alpha}$. Now to get the best rank between the nodes, by Lemma 2 we can write the rank between $X_{i}^{\alpha}$ and $X_{j}^{\alpha}$ as $W_{i j}^{\alpha}$ given as:

$$
W_{i j}^{\alpha}=n\left(P_{\alpha}\right)-n\left(X_{i}^{\alpha} \cap X_{j}^{\alpha}\right)
$$

where, $W_{i j}^{\alpha} \in W_{\alpha}$. Now we see that the value of $W_{i j}^{\alpha}$ should be less if the nodes are more similar, thus similar nodes shall have higher ranks between them.

In figure 1 , we have $W_{12}^{\alpha}=1, W_{23}^{\alpha}=3, W_{13}^{\alpha}=2, W_{43}^{\alpha}=4$, and $W_{14}^{\alpha}=5$. So the least score but higher rank is $W_{12}^{\alpha}=1$, which means the node $X_{1}^{\alpha}$ is very similar to node $X_{2}^{\alpha}$. The lowest rank among the nodes is $W_{14}^{\alpha}=5$, which means nodes $X_{1}^{\alpha}$ is not that similar to $X_{4}^{\alpha}$ in contrast to the node $X_{2}^{\alpha}$.

Now let us consider the unweighted situation, where we do not have to consider the similarity between nodes. Now we can re-write $W_{\alpha}$ as $E_{\alpha}$. We can then say that for the nodes $X_{\alpha}=\left\{X_{1}^{\alpha}, X_{2}^{\alpha}, X_{3}^{\alpha}, X_{4}^{\alpha}\right\}$ we can have $X_{\alpha} \times X_{\alpha}$ ordered pairs. Thus $E_{\alpha} \subset\left(\begin{array}{c}X_{\alpha} \\ 2\end{array}\right)$.

Now, the adjacency matrix of the layer $G_{\alpha}$ can be defined in two different ways based on whether it is weighted or unweighted. The adjacency matrix for layer $G_{\alpha}$ is represented as $A^{[\alpha]}=\left(a_{i j}^{\alpha}\right) \in \Re^{N_{\alpha} \times N_{\alpha}}$ given by:

For weighted network:

$$
\left(a_{i j}^{\alpha}\right)=\left\{\begin{array}{cc}
W_{i j}^{\alpha} & , \text { if }\left(X_{i}^{\alpha}, X_{j}^{\alpha}\right) \in E_{\alpha} \\
0 & , \text { otherwise }
\end{array}\right.
$$




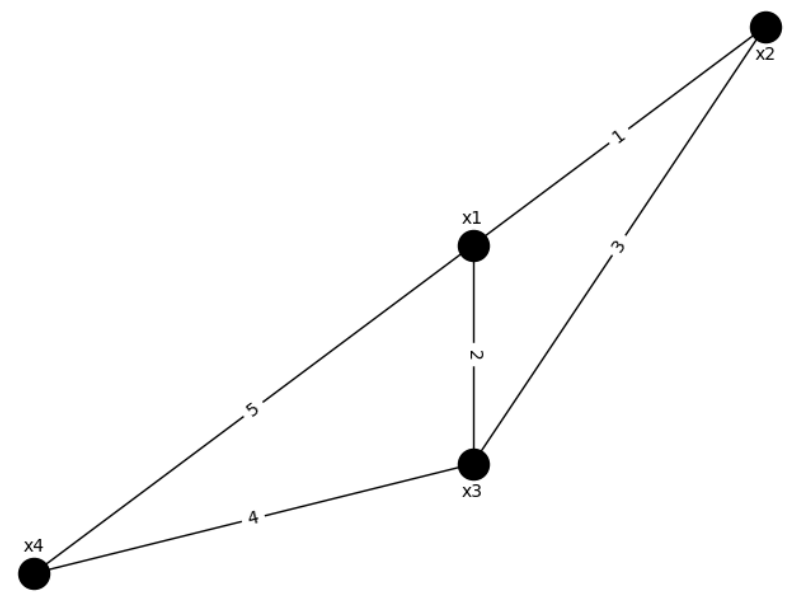

Figure 1: A sample plot of $G_{\alpha}$ layer

For unweighted network:

$$
\left(a_{i j}^{\alpha}\right)=\left\{\begin{array}{cc}
1 & , \text { if }\left(X_{i}^{\alpha}, X_{j}^{\alpha}\right) \in E_{\alpha} \\
0 & , \text { otherwise }
\end{array}\right.
$$

where $1 \leq i, j \leq N_{\alpha}$ and $1 \leq \alpha \leq M$. According to figure-1 $G_{\alpha}$ is weighted therefore its adjacency matrix can be given by:

$$
\left(a_{i j}\right)=\left(\begin{array}{llll}
0 & 1 & 2 & 5 \\
1 & 0 & 3 & 0 \\
2 & 3 & 0 & 4 \\
5 & 0 & 4 & 0
\end{array}\right)
$$

Proposition 1. Between any two layers $G_{\alpha}$ and $G_{\beta}, E_{\alpha \beta}$ exists if and only if $X_{i}^{\alpha} \in\left\{X_{j}^{\beta}\right\}$ holds true, irrespective whether the graph is weighted or unweighted, where $\alpha \neq \beta$.

Proof 4. In our relational network system, we know that $X_{i}^{\alpha}$ is any arbitrary node of $G_{\alpha}$ and $X_{i}^{\alpha}$ is a set of parameters where $X_{i}^{\alpha} \subseteq P_{\alpha}$, similarly $X_{j}^{\beta}$ is any arbitrary node of $G_{\beta}$ and $X_{j}^{\beta} \subseteq P_{\beta}$. Now let us consider a network situation where nodes of one layer are the parameter set of nodes of another layer. Let us perform a simple induction to see the above conditions. So, According to the figure 2 in layer $G_{\alpha}$ we have $E_{\alpha}=\{(2,3),(3,8),(10,0),(5,1), \ldots,(9,7)\}$ and $X_{\alpha}=\{0,1,2, . ., 14\}$, in layer $G_{\beta}$ we have $E_{\beta}=\{(3,2),(1,4)\}$ and $X_{\beta}=\{0,1,2,3,4\}$ here numbers represents the node names for example $X_{1}^{\alpha}=0$ and $X_{3}^{\beta}=2$ and so on. Now as mentioned earlier nodes of one layer are the parameter set of nodes of another layer, therefore, $X_{1}^{\beta}=\left\{X_{1}^{\alpha}, X_{3}^{\alpha}, X_{5}^{\alpha}\right\}$ here $X_{2}^{\beta}=\left\{X_{2}^{\alpha}=1\right\}$. So $E_{\alpha \beta}$ or the intra-layer edges can be given as $E_{i j}^{\alpha \beta} \in\left(X_{\alpha} \times X_{\beta}\right)$, therefore, $E_{22}^{\alpha \beta}=\left(X_{2}^{\alpha}, X_{2}^{\beta}\right)=(1,1)$ exists because, $X_{2}^{\alpha} \in\left\{X_{2}^{\beta}\right\}$. Again $E_{23}^{\alpha \beta}=\left(X_{2}^{\alpha}, X_{3}^{\beta}\right)=\phi$ does not exist as $X_{2}^{\alpha} \notin\left\{X_{2}^{\beta}\right\}$. Therefore for $E_{\alpha \beta}$ to exist in our network $X_{i}^{\alpha} \in\left\{X_{j}^{\beta}\right\}$ condition must satisfy. 


\section{Bi-Layer Network}

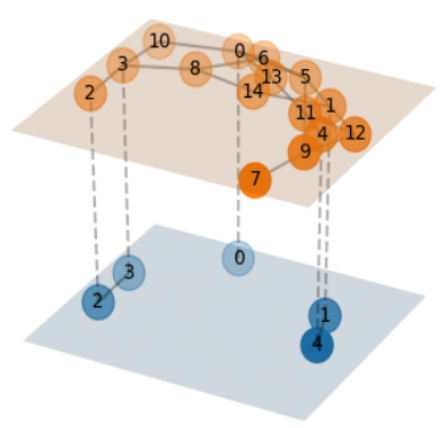

(a)

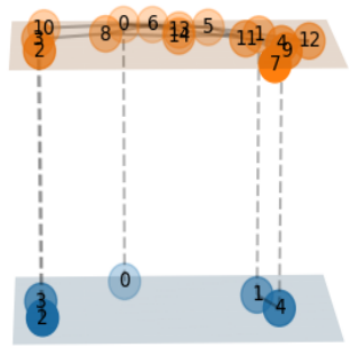

(b)

Figure 2: (a). Slanted view of the two layers $G_{\alpha}$ and $G_{\beta}$. (b). Intra-layer Edges $E_{\alpha \beta}$

Theorem 2. The weight of a relational multilayer network $\mathcal{M}=(\boldsymbol{\mathcal { Y }}, \mathfrak{J})$ is given as:

$$
W_{\alpha \beta}=\left\{\begin{array}{cc}
1 & \text {, if } \exists E_{\alpha \beta} \mid X_{i}^{\alpha} \in\left\{X_{j}^{\beta}\right\} \\
0 & \text {,otherwise }
\end{array}\right.
$$

and $W_{\alpha \beta} \in \mathfrak{J}$, where $1 \leq i \leq N_{\alpha}, 1 \leq j \leq N_{\beta}$ and $1 \leq \alpha, \beta \leq M$.

Corollary 2.2. The intra-layer adjacency matrix is given by $A^{[\alpha, \beta]}=\left(a_{i j}^{\alpha \beta}\right) \in \Re^{N_{\alpha} \times N_{\beta}}$ where,

$$
\left(a_{i j}^{\alpha \beta}\right)=\left\{\begin{array}{cc}
1 & , \text { if }\left(X_{i}^{\alpha}, X_{j}^{\beta}\right) \in E_{\alpha \beta} \\
0 & \text {, otherwise }
\end{array}\right.
$$

irrespective of being weighted or unweighted.

Proof 5. From Proposition 1 we can say that $E_{\alpha \beta}$ exists if and only if $X_{i}^{\alpha} \in\left\{X_{j}^{\beta}\right\}$. This $E_{\alpha \beta}$ is the intra-layer edges of the overall multilayer network $\mathcal{H}=(\boldsymbol{\mathcal { G }}, \mathcal{J})$. Now for each layers the weight between the nodes has been evaluated by means of similarity index as presented in Theorem 1. Between intra-layers, the weight has to be evaluated in a similar manner based on observability, where an element node of a layer is a parameter of the node of another layer. So, we can write:

$$
W_{\alpha \beta}=\left\{\begin{array}{cc}
1 & \text {, if } \exists E_{\alpha \beta} \mid X_{i}^{\alpha} \in\left\{X_{j}^{\beta}\right\} \\
0 & \text {,otherwise }
\end{array}\right.
$$

and $W_{\alpha \beta} \in$ J. For example, in figure 2 we can say the weight $W_{22}^{\alpha \beta}=(1,1)=1$ but $W_{74}^{\alpha \beta}=(6,3)=0$ because $E_{74}^{\alpha \beta}$ does not exist. Now similar to Corollary 1.2 the adjacency 
matrix of $\mathcal{M}$ is given by $A^{[\alpha, \beta]}=\left(a_{i j}^{\alpha \beta}\right) \in \Re^{N_{\alpha} \times N_{\beta}}$ where,

$$
\left(a_{i j}^{\alpha \beta}\right)=\left\{\begin{array}{cc}
1 & , \text { if }\left(X_{i}^{\alpha}, X_{j}^{\beta}\right) \in E_{\alpha \beta} \\
0 & , \text { otherwise }
\end{array}\right.
$$

irrespective whether the network is weighted or unweighted because $W_{\alpha \beta}$ can be only 0 and 1 similar to adjacency condition.

Lemma 3. The aggregate centrality of a node $X_{i}^{\alpha}$ between any two layers $G_{\alpha}$ and $G_{\beta}$ can be given as:

$$
\frac{1}{2} C_{i}^{\alpha}+\frac{1}{2} C_{i}^{\beta}
$$

where $C_{i}^{\alpha}$ represents the centrality measure of $X_{i}^{\alpha}$ in the layer $G_{\alpha}$ and $C_{i}^{\beta}$ represents the same in layer $G_{\beta}$ of $\mathcal{H}$.

Proof 6. Let us consider the layer $G_{\alpha}$, then the degree of node $X_{i}^{\alpha} \in X_{\alpha}$ is given by the relation:

$$
K_{i}^{[\alpha]}=\sum_{X_{i}^{\alpha} \in X_{\alpha}} \operatorname{deg}\left(X_{i}^{\alpha}\right)=2 n\left(E_{\alpha}\right)
$$

Now, for any node $\left.X_{i}^{\{1} 1, . ., \alpha, \ldots, M\right\}$ in any layer the degree becomes:

$$
K_{i}=\left\{K_{i}^{[1]}, \ldots, K_{i}^{[M]}\right\}
$$

we have $X_{i} \in X$, therefore the overlapping degree is given by:

$$
O_{i}=\sum_{\alpha=1}^{M} K_{i}^{[\alpha]}
$$

so, the eigenvector centrality can be written as $C_{\alpha}=\left(C_{1}^{[1]}, \ldots, C_{i}^{[\alpha]}, C_{N}^{[M]}\right)$, where $1 \leq \alpha \leq M$ in each layer. Now for every node the aggregate can be written as:

$$
\begin{gathered}
C^{[\alpha]}=\sum_{\alpha=1}^{M} C_{i}^{\alpha} \in \Re^{M} \\
C_{i}^{[\alpha]}=\frac{1}{2} C_{i}^{\alpha}
\end{gathered}
$$

Now, when we consider only a single layer we get half of the total centrality measure of the node for that layer. Hence, for two layers $G_{\alpha}$ and $G_{\beta}$ the aggregate centrality measure is given as $\frac{1}{2} C_{i}^{\alpha}+\frac{1}{2} C_{i}^{\beta}$ where $\alpha \neq \beta$. 
Theorem 3. The aggregate centrality measure of any node $X_{i} \in X$ in our relational multilayer network $\mathcal{M}$ with a total of ' $\mathrm{M}$ ' layers is given as:

$$
\sum_{\alpha=1}^{M} \frac{w_{\alpha}}{W} C_{i}^{\alpha}=\frac{1}{W} \sum_{\alpha=1}^{M} \sum_{\substack{\beta=1 \\ \beta \neq \alpha}}^{K} w_{\alpha \beta} C_{i}^{\alpha}
$$

where we weight the centrality measure of node $X_{i}$ in layer $G_{\alpha}$ by $w_{\alpha} / W$, where $w_{\alpha \beta}$ is the coupling strength (i.e. the weight of the intra-layer edges) between layers $G_{\alpha}$ and $G_{\beta}$ and $W=\sum_{\alpha=1}^{M} w_{\alpha}$ is a normalizing constant.

Proof 7. By extending Lemma-3 we now have the aggregate centrality measure for a node $X_{i}$ as $C_{i}=\sum_{\alpha=1}^{M} C_{i}^{\alpha}$. The strength of the intra-layer connection is given by $w_{\alpha \beta} \in \mathfrak{I}$ as per Theorem-2. So the intra-layer centrality measure depends upon the value of $w_{\alpha \beta}$, and is given by:

$$
\sum_{\alpha=1}^{M} \frac{w_{\alpha}}{W} C_{i}^{\alpha}=\frac{1}{W} \sum_{\alpha=1}^{M} \sum_{\substack{\beta=1 \\ \beta \neq \alpha}}^{K} w_{\alpha \beta} C_{i}^{\alpha}
$$

where, $W=\sum_{\alpha=1}^{M} w_{\alpha}$ becomes the normalizing constant for the above relation, and $1 \leq$ $\alpha, \beta \leq M$.

\section{Medical Trust-Network System}

In this section, We shall finally formulate the Trust-Network using the parameters Hospitals, Doctors, and the Departments with the help of the relational network theorems. So, we have three unique sets of Hospitals $\left(\mathrm{H}_{\mathrm{i}}\right)$, Doctors $\left(\mathrm{D}_{\mathrm{j}}\right)$, and $\operatorname{Departments}\left(\mathrm{P}_{\mathrm{k}}\right)$. So we define them as follows:

$$
\begin{aligned}
& H_{i}=\left\{h_{1}, h_{2}, \ldots, h_{i}\right\} \text { where } i \in \Re \\
& D_{j}=\left\{d_{1}, d_{2}, \ldots, d_{j}\right\} \text { where } j \in \Re \\
& P_{k}=\left\{p_{1}, p_{2}, \ldots, p_{k}\right\} \text { where } k \in \Re
\end{aligned}
$$

Now, for future correlation, we shall consider that sets $\mathrm{H}_{\mathrm{i}}, \mathrm{D}_{\mathrm{j}}$ and $\mathrm{P}_{\mathrm{k}}$ will have one to one correspondence with one another such that a relation ' $\mathbf{R}$ ' exists among them. Let us now design the individual layers of our multilayered n-partite graph

\section{Hospital Network}

In this layer, we will create a hospital network, such that each hospital is considered as a node of a graph $\mathbf{G}_{1}$, and its edges are defined by the count of similar departments they have between any two hospital nodes.

Now let us consider any two random hospitals say, ' $\alpha$ ' and ' $\beta$ '. Let $\mathrm{P}_{\alpha}$ be the set of departments in the hospital $\alpha$ and $\mathrm{P}_{\beta}$ be the set of departments in the hospital $\beta$. Now we know, since $\alpha$ and $\beta$ are part of the set $\mathrm{H}_{\mathrm{i}}$, then we can say $\mathrm{P}_{\alpha}$ and $\mathrm{P}_{\beta}$ is part of the universal set $\mathrm{P}_{\mathrm{k}}$. Now the weight or similarity between $\alpha$ and $\beta$ is given by the number of elements(here, 
departments) present in the intersection of the sets $\mathrm{P}_{\alpha}$ and $\mathrm{P}_{\beta}$. So, mathematically using the proposed Theorem 1 the edge weight between Hospital nodes ' $\alpha$ ' and ' $\beta$ ' is given by:

$$
W_{\alpha \beta}=n\left(P_{k}\right)-n\left(P_{\alpha} \cap P_{\beta}\right) \quad \forall \alpha, \beta \in H_{i} \text { and } P_{\alpha}, P_{\beta} \in P_{k}
$$

Where, $\mathrm{P}_{\alpha}$ and $\mathrm{P}_{\beta}$ are set of departments in hospitals $\alpha$ and $\beta$ respectively, and $\mathrm{P}_{\alpha}$ and $\mathrm{P}_{\beta}$ are subsets of $\mathrm{P}_{\mathrm{k}}$, which is a universal set containing the list of all possible departments.

\section{Department Network}

In this layer, we will create the department network, such that each department is now considered as a node of another graph $\mathbf{G}_{2}$, and its edges are defined by the count of similar doctors working in different department nodes.

Now let us consider any two random departments say, ' $r$ ' and ' $t$ '. Let $D_{\mathrm{r}}$ be the set of doctors working in the department $r$ and $D_{t}$ be the set of doctors working in the department $t$. Now we know, since $r$ and $t$ are part of the set $P_{k}$, then we can say $D_{r}$ and $D_{t}$ is part of the universal set $D_{j}$. Now the weight or similarity between $r$ and $t$ is given by the number of elements(here, doctors) present in the intersection of the sets $D_{r}$ and $D_{t}$. So, mathematically using the proposed Theorem 1 the edge weight between Department nodes ' $r$ ' and ' $t$ ' is given by:

$$
W_{r t}=n\left(D_{j}\right)-n\left(D_{r} \cap D_{t}\right) \quad \forall r, t \in P_{k} \text { and } D_{r}, D_{t} \in D_{j}
$$

Where $D_{r}$ and $D_{t}$ are sets of doctors working in departments $r$ and t respectively, and $D_{r}$ and $\mathrm{D}_{\mathrm{t}}$ are subsets of $\mathrm{D}_{\mathrm{j}}$, which is a universal set containing the list of all possible doctors.

\section{Doctor Network}

In this layer, we will create the doctor network, such that each doctor is now considered as a node of another graph $\mathbf{G}_{3}$, and its edges are defined by the count of similar workplaces, here hospitals, they work in.

Now let us consider any two random doctors say, 'p' and ' $q$ '. Let $\mathrm{H}_{\mathrm{p}}$ be the set of Hospitals doctor ' $\mathrm{p}$ ' work in and $\mathrm{H}_{\mathrm{q}}$ be the set of Hospitals doctor ' $\mathrm{q}$ ' work in. Now we know, since $\mathrm{p}$ and $q$ are part of the set $\mathrm{D}_{\mathrm{j}}$, then we can say $\mathrm{H}_{\mathrm{p}}$ and $\mathrm{H}_{\mathrm{q}}$ is part of the universal set $\mathrm{H}_{\mathrm{i}}$. Now the weight or similarity between $\mathrm{p}$ and $\mathrm{q}$ is given by the number of elements(here, hospitals) present in the intersection of the sets $\mathrm{H}_{\mathrm{p}}$ and $\mathrm{H}_{\mathrm{q}}$. So, mathematically using the proposed Theorem 1 the edge weight between Doctor nodes ' $p$ ' and ' $q$ ' is given by:

$$
W_{p q}=n\left(H_{i}\right)-n\left(H_{p} \cap H_{q}\right) \quad \forall p, q \in D_{j} \text { and } H_{p}, H_{q} \in H_{i}
$$

Where $\mathrm{H}_{\mathrm{p}}$ and $\mathrm{H}_{\mathrm{q}}$ are sets of hospitals where doctors $\mathrm{p}$ and $\mathrm{q}$ work on respectively, and $\mathrm{H}_{\mathrm{p}}$ and $\mathrm{H}_{\mathrm{q}}$ are subsets of $\mathrm{H}_{\mathrm{i}}$, which is a universal set containing the list of all possible Hospitals.

\section{Intra-layer Network}

In this section, we shall establish the intra-layer connection between each layer $\mathbf{G}_{1}, \mathbf{G}_{2}$, and $\mathbf{G}_{3}$. According to Theorem 2, we can say that for a random Doctor $\mathrm{P}$ he can either work in 


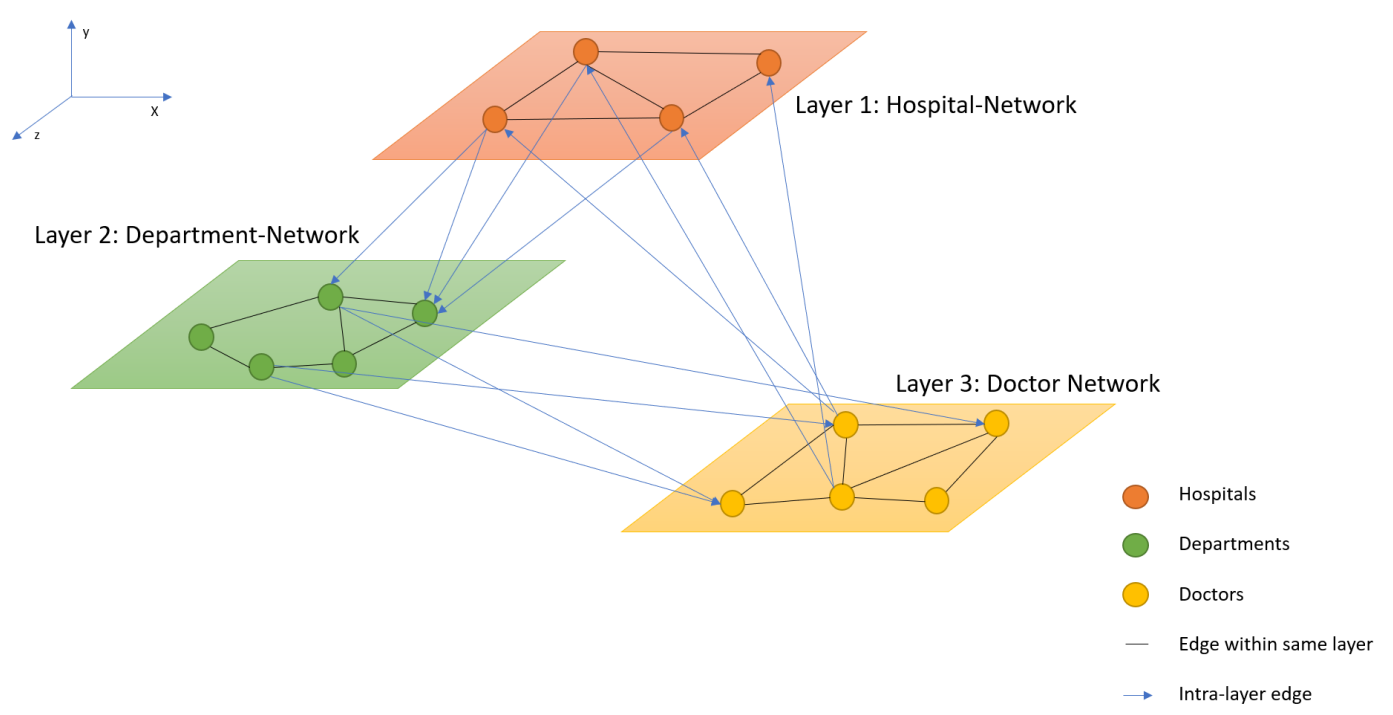

Figure 3: A slanted view of three layered Medical Trust-Network $G_{1}, G_{2}$, and $G_{3}$

any random Department $\mathrm{Q}$ on any random Hospital $\mathrm{R}$ or he does not work in Department $\mathrm{Q}$ on any random Hospital $\mathrm{R}$. So the intra-layer edge weight $W_{\alpha \beta} \in \mathcal{M}$ is 0 or 1 between any two layers of $G_{1}, G_{2}$, and, $G_{3}$. Here, $\mathcal{M}$ represents the overall Trust Network and it is a Relational Multilayered Network System. Figure 3 shows a rough model of our multilayered trust network.

\section{Discussion}

Our multilayered trust network is based on the key observable characteristics of any medical healthcare system. Formulation of such complex networks has been traditionally derived from bipartite graph systems, where two dissimilar sets are related. The mathematical implementation of these kind of networks has been done in many papers like (Domenico et al.)[31],(Scabini et al.)[32], (Boccaletti et al.)[33], and many others. The structural architecture is some-what similar to the model proposed by (Scabini et al.) [32] in his paper in which he designed a multilayered complex network to explain the color-texture characterization $\{\mathrm{R}, \mathrm{G}, \mathrm{B}\}$.

The centrality measure as expressed in theorem 3 of our network model is a generalised measure which incorporates betweeness, closeness, and degree centrality as proposed by (Kivelä et al.)[34]. The build of our network is greatly influenced by barabasi models to map the relation between disease and symptoms based on genotypes and phenotypes(observable characteristics) put forward in his paper [35].

Our model consists of multilayered interaction between the network entities unlike the previous feed-forward model proposed by (Guo et al.)[29], thus reducing the computational complexity. Due to this, our model also shows all possible interactions between the network entities, namely - doctors, their departments, and their workplace hospitals or medical centers. Though our model has discrete relationship between its entities, yet we have kept our 
model malleable so that we can work on the stochastic nature of these entities. Valles-Catala et al.[36] and Levent et al.[3] explores how stochastic block models or SBMs can be used to reveal the multilayered structure on any complex network. If we can introduce stochastic or probabilistic functions to describe the interactions between our network entities we will certainly be one step closer in modeling a scale-free network, which is considered elegant and versatile.

\section{Conclusion and Future Work}

We have proposed a medical Trust Network model using multilayered complex network. Our approach relied on the observable characteristics like doctor names, departments and hospital records to model discrete relationship between these entities. Our proposed centrality measure can be used to study the nature of our multilayered network in much more depth. This centrality measure is derived from n-partite multilayered graph system. We validated our model by calculating its computational complexity, which we have found to be much better than the previously proposed models. Although our multilayered network model has been designed considering there exist discrete relationships between medical entities, however we could possibly use other relationships like stochastic functions to describe such interactions. Finally, the proposed Trust-Network can be explored further by studying the nature of stochastic or probabilistic interactions between the medical entities using scale-free random models. 


\section{Bibliography}

[1] Gopichandran V, Chetlapalli SKFactors influencing trust in doctors: a community segmentation strategy for quality improvement in healthcareBMJ Open 2013;3:e004115. doi: 10.1136/bmjopen-2013-004115

[2] Yuan, W., Guan, D., Lee, Y. et al. The small-world trust network. Appl Intell 35, 399-410 (2011). https://doi.org/10.1007/s10489-010-0230-7

[3] Levent V. Orman. 2013. Bayesian Inference in Trust Networks. ACM Trans. Manage. Inf. Syst. 4, 2, Article 7 (August 2013), 21 pages. DOI:https://doi.org/10.1145/2489790

[4] Artz D, Gil Y (2007) A survey of trust in computer science and the Semantic Web. Web Semant 5:58-71

[5] O'Donovan J, Smyth B (2005) Trust in recommender systems. In: Proceedings of the 10th international conference on intelligent.

[6] Magnier-Watanabe, Rémy, Michiko Yoshida, and Tomoaki Watanabe. "Social network productivity in the use of SNS." Journal of knowledge management (2010).

[7] Coates G, Hopkinson K, Graham S, Kurkowski S (2008) Collaborative, trust-based security mechanisms for a regional utility intranet. IEEE Trans Power Syst 23:831-844

[8] Avijit Gayen, Maitry Bhavsar, and Joydeep Chandra. 2017. Towards a Social Trust Based Measure of Scientific Productivity. In Proceedings of ASONAM '17, Sydney, Australia, July 31-August 03, 2017, 8 pages. https://doi.org/10.1145/3110025.3110082

[9] Igarashi, Tasuku, et al. "Culture, trust, and social networks." Asian Journal of Social Psychology 11.1 (2008): 88-101.

[10] Barabási, Albert-László, Réka Albert, and Hawoong Jeong. "Mean-field theory for scalefree random networks." Physica A: Statistical Mechanics and its Applications 272.1-2 (1999): 173-187.

[11] Adamic LA (1999) The small world web. In: Proceedings of the third European conference on research and advanced technology for digital libraries. Springer, Berlin, pp $443-452$

[12] Barabási, Albert-László, Réka Albert, and Hawoong Jeong. "Scale-free characteristics of random networks: the topology of the world-wide web." Physica A: statistical mechanics and its applications 281.1-4 (2000): 69-77. 
[13] Andaleeb, Syed Saad. "Service quality perceptions and patient satisfaction: a study of hospitals in a developing country." Social science medicine 52.9 (2001): 1359-1370.

[14] Ziqi, T. All-Round Marketing Increases Hospital Popularity. Cell Biochem Biophys 72, 367-370 (2015). https://doi.org/10.1007/s12013-014-0468-5

[15] Gonzalez, M. L., and J. A. Rizzo. 1991. "Physician Referrals and the Medical Market Place." Medical Care 29 (10): 1017-27.

[16] Keating, N. L., A. M. Zaslavsky, and J. Z. Ayanian. 1998. "Physicians' Experiences and Beliefs Regarding Informal Consultation." Journal of American Medical Association 280 (10): 900-4.

[17] Gabbay, J., and A. le May. 2004. "Evidence Based Guidelines or Collectively Constructed "Mindlines?" Ethnographic Study of Knowledge Management in Primary Care." British Medical Journal 329 (7473): 1013-7.

[18] Barnett, M. L., Landon, B. E., O’Malley, A. J., Keating, N. L., Christakis, N. A. (2011). Mapping physician networks with self-reported and administrative data. Health services research, 46(5), 1592-1609. https://doi.org/10.1111/j.1475-6773.2011.01262.x

[19] Davies, H. T., Lampel, J. (1998). Trust in performance indicators?. BMJ Quality Safety, $7(3), 159-162$.

[20] Pearson, S.D. and Raeke, L.H. (2000), Patients' Trust in Physicians: Many Theories, Few Measures, and Little Data. Journal of General Internal Medicine, 15: 509-513. doi:https://doi.org/10.1046/j.1525-1497.2000.11002.x

[21] Calnan, M. W., Sanford, E. (2004). Public trust in health care: the system or the doctor?. BMJ Quality Safety, 13(2), 92-97.

[22] Iyengar, R., Van den Bulte, C., Valente, T. W. (2011). Opinion leadership and social contagion in new product diffusion. Marketing Science, 30(2), 195-212.

[23] Christakis, N. A., Fowler, J. H. (2011). Commentary - contagion in prescribing behavior among networks of doctors. Marketing Science, 30(2), 213-216.

[24] Newman, M. E. (2003). The structure and function of complex networks. SIAM review, $45(2), 167-256$.

[25] Hewett D.G., Watson B.M., Gallois C. (2013) Trust, distrust, and communication accommodation among hospital doctors. In: Candlin C.N., Crichton J. (eds) Discourses of Trust. Palgrave Studies in Professional and Organizational Discourse. Palgrave Macmillan, London

[26] Chambers, D., Wilson, P., Thompson, C., Harden, M. (2012). Social network analysis in healthcare settings: a systematic scoping review. PloS one, 7(8), e41911. https://doi.org/10.1371/journal.pone.0041911 
[27] Keating, N. L., Ayanian, J. Z., Cleary, P. D., Marsden, P. V. (2007). Factors affecting influential discussions among physicians: a social network analysis of a primary care practice. Journal of general internal medicine, 22(6), 794-798.

[28] Guo, L., Jin, B., Yao, C., Yang, H., Huang, D., Wang, F. (2016). Which doctor to trust: a recommender system for identifying the right doctors. Journal of medical Internet research, 18(7), e186

[29] Chakraborty, S., Gayen, A. (2020, May 24). Discrete nature of relational trust networks: A multilayered model for healthcare systems. https://doi.org/10.35543/osf.io/7wnyz.

[30] Sharara H, Getoor L, Norton M. Active surveying: a probabilistic approach for identifying key opinion leaders. 2011 Presented at: The Twenty-Second international joint conference on Artificial Intelligence (IJCAI'11); July 16-22, 2011; Barcelona, Spain p. 1485-1490.

[31] De Domenico, M., Solé-Ribalta, A., Cozzo, E., Kivelä, M., Moreno, Y., Porter, M. A., ... Arenas, A. (2013). Mathematical formulation of multilayer networks. Physical Review X, 3(4), 041022.

[32] Scabini, L. F., Condori, R. H., Gonçalves, W. N., Bruno, O. M. (2019). Multilayer complex network descriptors for color-texture characterization. Information Sciences, 491, 30-47.

[33] Boccaletti, S., Bianconi, G., Criado, R., Del Genio, C. I., Gómez-Gardenes, J., Romance, M., ... Zanin, M. (2014). The structure and dynamics of multilayer networks. Physics Reports, 544(1), 1-122.

[34] Mikko Kivelä, Alex Arenas, Marc Barthelemy, James P. Gleeson, Yamir Moreno, Mason A. Porter, Multilayer networks, Journal of Complex Networks, Volume 2, Issue 3, September 2014, Pages 203-271, https://doi.org/10.1093/comnet/cnu016

[35] Zhou, X., Menche, J., Barabási, A. L., Sharma, A. (2014). Human symptoms-disease network. Nature communications, 5(1), 1-10.

[36] Valles-Catala, T., Massucci, F. A., Guimera, R., Sales-Pardo, M. (2016). Multilayer stochastic block models reveal the multilayer structure of complex networks. Physical Review X, 6(1), 011036. 